\title{
Generalizations of Rough Functions in Topological Spaces by Using Pre-Open Sets
}

\author{
Amgad S. Salama1, Hassan M. Abu-Donia ${ }^{2}$ \\ ${ }^{1}$ Department of Mathematics, Faculty of Science, Tanta University, Tanta, Egypt; ${ }^{2}$ Department of Mathematics, Faculty of Science, \\ Zagazig University, Zagazig, Egypt. \\ Email: \{dr_salama75, donia_1000\}@yahoo.com
}

Received January $25^{\text {th }}, 2011$; revised March $6^{\text {th }}, 2012$; accepted March $14^{\text {th }}, 2012$

\begin{abstract}
Functions are a means to link or transport from a world to another world may be similarly or completely different from the other world. In this paper we addressed the issue of rough functions and the possibility of transfer it from the real line to the topological abstract view that can be applied to intelligent information systems. The rough function approach has not been studied much specially from a topological point of view. Here we developed a new type of topological generalizations of rough functions with reference to how it is used in medical applications. Considering that the function is in the original a relation can be based on a review of all circular functions from the perspective of relations. Accordingly, the dream that the generalizations of rough functions are transferred to all papers prior to a comprehensive computer application.
\end{abstract}

Keywords: Rough Sets; Rough Numbers; Approximation Spaces; Topological Spaces; Fuzzy Sets

\section{Introduction}

Rough set theory [1], is an extension of set theory for the study of intelligent systems characterized by inexact, uncertain or insufficient information. Moreover, this theory may serve as a new mathematical tool to soft computing besides fuzzy set theory [2-4], and has been successfully applied in machine learning, information sciences, expert systems, data reduction, and so on. Recently, lots of researchers are interested to generalize this theory in many fields of applications [5-7]. In classical rough set theory, partition or equivalence (indiscernibility) relation is an important and primitive concept. But, partition or equivalence relation is still restrictive for many applications. To study this issue, several interesting and meaningful generalizations to equivalence relation have been proposed in the past, such as tolerance relations [8], topological bases and subbases [9-12]. Particularly, some researchers have used coverings of the universe of discourse for establishing the generalized rough sets by coverings [13]. Others [14-16] combined fuzzy sets with rough sets in a fruitful way by defining rough fuzzy sets and fuzzy rough sets. Furthermore, another group has characterized a measure of roughness of a fuzzy set making use of the concept of rough fuzzy sets [17-19]. They also suggested some possible real world applications of these measures in pattern recognition and image analysis problems. Some results of these generalizations are obtained about rough sets and fuzzy sets in [20-22].

Topological ideas are present not only in almost all areas of today mathematics, for example biochemistry [23] information systems [24] and others for more fields of topology applications [25] and its related links. The subject of topology itself consists of several different branches such as point set topology, algebraic topology and differential topology which have relatively little in common this richness of applications and differentiate between branches of topology implied a difficult to give an accurate definition for topology. The topology concepts like continuity, irresoluteness, compactness, connectedness, convergence, denseness and others are as basic to mathematicians. The topology structure $\tau$ on a set $X$ is a general tool for constructing the above concepts. This tool contains many classes of near open sets such as: regular open [26], semi open sets [27], pre-open sets [28], $\beta$-open sets [29] and $b$-open sets [30]. Many authors used the previous types of near open sets to introduce some types of near continuous functions such as: In [28] the concept of pre-continuous functions are introduced. In [31] the concept of $\alpha$-continuous functions is introduced.

The pair of lower and upper approximation operators is just a pair of interior and closure operators of a topology [32-34]. In [35] the concept of rough functions is introduced. In $[35,36]$ we found the definition of the rough real number. In this paper, we propose to give a further 
study on rough functions and to introduce some concepts based on rough functions. In the beginning we will study rough sets on the real line.

In Section 2, we will initiate the notion of rough real functions. The aim of Section 3 is to define and study the new notion of "topological pre-rough function". The main goal of Section 4 is to initiate and study the pre-approximations of a function as a relation. Finally, we aim in Section 5 to define an alternative description of the topological pre-rough functions and topological pre-rough continuity.

A topological space [36] is a pair $(X, \tau)$ consisting of a set $X$ and family $\tau$ of subsets of $X$ satisfying the following conditions:

1) $\varphi, X \in \tau$.

2) $\tau$ is closed under arbitrary union.

3) $\tau$ is closed under finite intersection.

The pair $(X, \tau)$ is called a topological space, the elements of $X$ are called points of the space, the subsets of $X$ belonging to are called open set in the space, and the complement of the subsets of $X$ belonging to $\tau$ be called closed set in the space; the family $\tau$ of open subsets of $X$ is also called a topology for $X$.

$$
\bar{A}=\bigcap\left\{F \subseteq X: A \subseteq F, F \in \tau^{c}\right\} \text { is called } \tau \text {-closure of }
$$
a subset $A \subset X$.

Evidently, $A$ is the smallest closed subset of $X$ which contains $A$. Note that $A$ is closed iff $A=\bar{A}$.

$A^{\circ}=\bigcup\{G \subseteq X: G \subseteq A, G \in \tau\}$ is called the $\tau$-interior of a subset $A \subseteq X$.

Evidently, $A^{\circ}$ is the union of all open subsets of $X$ which containing in $A$. Note that $A$ is open iff $A=A^{\circ}$. And $b(A)=\bar{A}-A^{\circ}$ is called the $\tau$-boundary of a subset $A \subseteq X$.

Let $A$ be a subset of a topological space $(X, \tau)$. Let $\bar{A}, A^{\circ}$ and $b(A)$ be closure, interior, and boundary of $A$ respectively. $A$ is exact if $b(A)=\varphi$, otherwise $A$ is rough. It is clear $A$ is exact iff $\bar{A}=A^{\circ}$.

Definition 1.1: A subset $A$ of a topological space $(X, \tau)$ is called pro-open if $A \subset \operatorname{int}(\operatorname{cl}(A))$.

The family of all pre-open sets of $X$ is denoted by $P O(X)$. The complement of pre-open set is preclosed set. The family of preclosed sets is denoted by $P C(X)$.

Definition 1.2: A function $f:(X, \tau) \rightarrow(Y, \sigma)$ is said to be pre-continuous if $f^{-1}(G) \in P O(X)$ for every $G \in \sigma[28]$.

\section{Rough Functions on Real Line}

Let $R^{+}$be the set of non-negative real numbers, and let $S e q \subseteq R^{+}$be a sequence of real numbers defined by $x_{1}, x_{2}, x_{3}, \cdots, x_{n}, \cdots$ such that $x_{1}<x_{2}<x_{3}<\cdots<x_{n}<\cdots$. The sequence $\mathrm{Seq}$ defines the partition $\pi(\mathrm{Seq})$ of $R^{+}$ by $\pi(S e q)=\left\{0,\left(0, x_{1}\right), x_{1},\left(x_{1}, x_{2}\right), x_{2}, \cdots,\left(x_{i}, x_{i+1}\right), \cdots\right\}$, where $\left(x_{i}, x_{i+1}\right)$ denote open intervals $R^{+}$. The sequence $S e q$ is called a categorization of $R^{+}$and the ordered pair $A=\left(R^{+}, \pi^{*}(S e q)\right)$ is an approximation space, where $\pi^{*}(\mathrm{Seq})$ is the equivalence relation associated with $\pi(S e q)$.

Let $A=\left(R^{+}, \pi^{*}(S e q)\right)$ be an approximation space. By $\operatorname{Seq}(x)$ in $A$ we denote the block of the partition $\pi(\operatorname{Seq})$ containing $x$, in particular if $x \in S e q$, we have $\operatorname{Seq}(x)=\{x\}, \operatorname{clSeq}(x)$ is the closure of $\operatorname{Seq}(x)$ with respect to the usual topology on $R$. Let $A=\left(R^{+}, \pi^{*}(\operatorname{Seq})\right)$ be an approximation space, by $Q(x)$ we denote the closed interval $[0, x]$ for $x \in R^{+}$. For any $x \in R^{+}$, the Seq-lower and the Seq-upper approximations of $Q(x)$ in the approximation space $A=\left(R^{+}, \pi^{*}(S e q)\right)$ are defined respectively by

$$
\begin{aligned}
& \operatorname{Seq}{ }_{*}[Q(x)]=\left\{y \in R^{+}: \operatorname{Seq}(y) \subseteq Q(x)\right\} \\
& \operatorname{Seq}[Q(x)]=\left\{y \in R^{+}: \operatorname{Seq}(y) \cap Q(x) \neq \varphi\right\}
\end{aligned}
$$

The approximations of the closed interval $Q(x)=[0, x]$ can be understood as the approximations of the real number $x$ which are simply the ends of the interval $\operatorname{Seq}(x)$. The number $x$ is a rough number if

$\operatorname{Seq}_{*}[Q(x)] \neq \operatorname{Seq}^{*}[Q(x)]$, otherwise it is an exact number.

Example 2.1: Let $R^{+}$be the set of all non-negative real numbers, and let $N=\{1,2,3, \cdots\}$ be the set of natural numbers to be a sequence in $R^{+}$. Then the partition induced by $N$ is

$\pi(N)=\{0,(0,1), 1,(1,2), 2, \cdots, n,(n, n+1), n+1, \cdots\}$ and hence, $A=\left(R^{+}, \pi^{*}(N)\right)$ is an approximation space. Also, for any number $x \in N$, we have $N(x)=\{x\}$ and for any $x \notin N, N(x)=\left(x_{i}, x_{i+1}\right)$ and $x \in\left(x_{i}, x_{i+1}\right)$, Then every number $x \notin N$ is a rough number in $A$.

According to Example 1, we followed the following steps to get the approximations of a number $x$, say $x=1.2$. We remark that the required approximations of $x=1.2$ can be obtained directly in one step by $\operatorname{Bnd}(Q(1.2))=N(1.2)$.

Let $X$ and $Y$ be two subsets of $R^{+}$, and let $A=(X, S)$ and $B=(Y, P)$ be two approximation spaces, where $S$ and $P$ are equivalence relations on $X$ and $Y$, respectively, $f: X \rightarrow Y$ is a function. Then we define $(S, P)$-lower approximation of $f$ as the function $f_{*}: X \rightarrow Y$, such that $f_{*}(x)=P_{*}[f(x)]$ for every $x \in X$, and $(S, P)$ -upper approximation of $f$ as the function $f^{*}: X \rightarrow Y$, such that $f^{*}(x)=P^{*}[f(x)]$, for every $x \in X$.

We see that the term $(S, P)$ in the above definition can be replaced by $P$ only since the approximations of the function $f$ depends only on $P$.

Let $f: X \rightarrow Y$ be a real valued function, where $X$ and $Y$ are two subsets of $R^{+}$. The function $f$ is called a rough function at a point $x \in X$ if and only if $f_{*}(x) \neq f^{*}(x)$ and $f$ is called a rough function on $X$ if it is a rough function at every point $x \in X$.

We give the following example to indicate the above notions.

Example 2.2: Let $f: R^{+} \rightarrow R^{+}$be a real valued fun- 
ction defined by $f(x)=x+1$ for every $x \in R^{+}$. We denote the odd and even integers by $O$ and $E$, respectively, then $A=\left(R^{+}, \pi^{+}(O)\right)$ and $B=\left(R^{+}, \pi^{*}(E)\right)$ are approximation spaces, where $\pi(O)$ and $\pi(E)$ are partitions of $R^{+}$defined by $\pi(O)=\{0,(0,1), 1,(1,3), \cdots\}$ and $\pi(E)=\{0,(0,1), 1,(1,3), \cdots\}$, then at every point $x \in R^{+}$we define $E$-lower approximation of $f$ by $f_{*}: R^{+} \rightarrow R^{+}$such that

$$
\begin{aligned}
f_{*} & =E_{*}[f(x)]=E_{*}[x+1]=E_{*}[0, x+1] \\
& =\left\{y \in R^{+}: E(y) \subseteq[0, x+1]\right\}
\end{aligned}
$$

and the $E$-upper approximation of $f$ by the function $f^{*}: R^{+} \rightarrow R^{+}$such that

$f_{*}(x)=E^{*}[f(x)]=\left\{y \in R^{+}: E(y) \cap[0, x+1] \neq \varphi\right\}$.

For $x=3$, we have $f(3)=3+1=4$, then

$$
f_{*}(3)=E_{*}(4)=E_{*}([0,4])
$$

$=\left\{y \in R^{+}: E(y) \subseteq[0,4]\right\}=[0,4]$

$$
=\left\{y \in R^{+}: E(y) \cap[0,4] \neq \varphi\right\}=[0,4] \text {. }
$$

and $f^{*}(3)=E^{*}(4)=E^{*}([0,4])$. Then $f$ is an exact function at $x=3$, similarly we can prove that $f$ is an exact functional at every odd natural number.

For $x=2$, then

$$
\begin{aligned}
f_{*}(2) & =E_{*}(3)=E_{*}([0,3]) \\
& =\left\{y \in R^{+}: E(y) \subseteq[0,3]\right\}=[0,2]
\end{aligned}
$$

But

$$
\begin{aligned}
f^{*}(2) & =E^{*}(3)=E^{*}([0,3]) \\
& =\left\{y \in R^{+}: E(y) \cap[0,3] \neq \varphi\right\}=[0,4]
\end{aligned}
$$

Then $f$ is a rough function at $x=2$, similarly it can be proved that $f$ is a rough function at every even natural number.

Also, we notice that $f$ is a rough function at every $x \notin N$. Then $f$ is a rough function at every point $x \notin N$ or $x$ is an even natural number.

Let $f: X \rightarrow Y$ be a real valued function. Then $f$ is called $(S, P)$-continuous (roughly continuous) at a point $x \in X$ if $f[c l S(x)] \subseteq P^{*}([f(x)])$, where $A=(X, S)$ and $B=(Y, P)$ are approximation spaces.

Let $f: X \rightarrow Y$ be a real valued function. Then $f$ is roughly continuous on $X$ if $f$ is a roughly continuous at every point $x \in X$.

Example 2.3: According to Example 2, the function $f: R^{+} \rightarrow R^{+}$is a rough function at $x=1.5$ but $f(\operatorname{cls}(1.5))=[2,4]$ and $P^{*}(f(1.5))=E^{*}[2.5]=[0,4)$, then $f$ is not a rough continuous function at the rough number $x=1.5$, but at $x=1$, since $f(\operatorname{cls}(1))=\{2\}$ and $E^{*}(f(1))=[0,2]$ then $f$ is a roughly continuous at $x=1$, also at every $x \in N$ such that $x$ is odd number $f$ is roughly continuous. If $x$ is an even number, then $f$ is not a roughly continuous; hence $f$ is not a roughly continuous function on $R^{+}$.
Example 2.4: Let $X$ and $Y$ be subsets of $R^{+}$, such that $X=\{1,3,5,7\}$ and $Y=\{2,4,6\}$ and the real valued function $f: X \rightarrow Y$ be defined by $f(1)=f(5)=2$, $f(3)=4$ and $f(7)=6$, and consider the approximation spaces $A=(X, S)$ and $B=(Y, P)$, where $X \backslash S=\{\{1,5\},\{3,7\}\}$ and $Y \backslash P=\{\{2\},\{4,6\}\}$ we define the function $f_{*}: X \rightarrow Y$ by $f_{*}(x)=P_{*}(f(x))$. Then,

$$
\begin{array}{ll}
f_{*}(1)=P_{*}(2)=\{2\}, & f_{*}(3)=P_{*}(4)=\varphi, \\
f_{*}(5)=P_{*}(2)=\{2\}, & f_{*}(7)=P_{*}(6)=\varphi .
\end{array}
$$

Also for the function $f^{*}: X \rightarrow Y$ such that $f^{*}(x)=P^{*}(f(x))$. Then, $f^{*}(1)=P^{*}(2)=\{2\}$, $f^{*}(3)=P^{*}(4)=\{4,6\}, f^{*}(5)=P^{*}(2)=\{2\}$, $f^{*}(7)=P^{*}(6)=\{4,6\}$. Then the function $f$ is $P$-rough at $x=3.7$ and $f$ is not $P$-rough function at $x=1.5$.

Now, if $x=1$, then $\operatorname{Seq}(x)=\operatorname{clSeq}(x)=\{1,5\}$ and we have $f(\operatorname{clSeq}(x))=f(\{1,5\})=\{2\}$ and $P^{*}(f(x))=P^{*}(\{2\})=\{2\}$,

then $f(\operatorname{clSeq}(x)) \subseteq P^{*}(f(x))$, i.e., the function $f$ is $(S, P)$-roughly continuous at $x=1$.

If $x=3$, then $\operatorname{Seq}(x)=\operatorname{clSeq}(x)=\{3,7\}$ and $f(\{3,7\})=\{4,6\}$, but $P^{*}(f(x))=P^{*}(\{4\})=\{4,6\}$ then, the function $f$ is $(S, P)$-roughly continuous at $x=3$. Also at $x=5.7$ we find that $f$ is $(S, P)$-roughly continuous, hence $f$ is $(S, P)$-roughly continuous on $X$.

\section{Topological Pre-Rough Functions}

We purpose to generalize the concept of rough function to topological pre-rough function by using pre-open sets in topological spaces. Let $(X, \tau)$ be a topological space and $x \in X$. Then $\operatorname{pmin}(x)=\bigcap\{G \in P O(X): x \in G\}$ is called the pre minimal set containing the point $x$ with respect to pre-open sets in the topology $\tau$ on $X$.

The principle topology on a set $X$ is the topology has the minimal bases that consists only of minimal open sets at each $x \in X$.

Theorem 3.1: A topology $\tau$ on a set $X$ is principle iff arbitrary intersections of members of $\tau$ are members of $\tau$ [20].

Let $(X, \tau)$ be a principle topological space, for any element $x \in X$, we define pre-sequence by the set $\operatorname{Seq}(x)=\bigcap\{G: G \in P O(X), x \in G\}$ and by ${ }^{p} \overline{\operatorname{Seq}}(x)$ we mean the pre-closure of $\operatorname{Seq}(x)$ in $(X, \tau)$.

If $f:(X, \tau) \rightarrow(Y, \sigma)$ is a function between principle spaces $(X, \tau)$ and $(Y, \sigma)$, we define the functions $\operatorname{pmin}_{f}:(X, \tau) \rightarrow(Y, \sigma)$, by

$\operatorname{pmin}_{f}(x)=\bigcap\left\{G^{\prime}: G^{\prime} \in P O(Y)\right.$ and $f(x) \in G^{\prime}$ for every $\{x \in X\}$, and $p_{f}:(X, \tau) \rightarrow(Y, \sigma)$, by $\operatorname{pcl}_{f}(x)=\operatorname{pcl}_{\sigma}(f(x))$ for all $x \in X$.

Let $f:(X, \tau) \rightarrow(Y, \sigma)$ be a function, where $X$ and $Y$ are principle spaces. The function $f$ is called a topological pre-rough function at the point $x$ in $X$ if and only if $\operatorname{pmin}_{f}(x) \neq p_{f}(x)$, and $f$ is a topological pre-rough 
function on $X$ if it is a topological pre-rough function at every point $x$ in $X$.

Example 3.1: Let $(X, \tau)$ and $(Y, \sigma)$ be topological spaces, where $X=\{a, b, c, d\}$,

$\tau=\{X, \varphi,\{a\},\{c\},\{d\},\{a, c\},\{a, d\},\{c, d\},\{a, c, d\}\}$ and $Y=\{1,2,3,4\}, \sigma=\{Y, \varphi,\{1\},\{2\},\{1,2\},\{1,2,3\}\}$.

Let $f:(X, \tau) \rightarrow(Y, \sigma)$ be a map defined by $f(a)=3$, $f(b)=1, f(c)=4$ and $f(d)=2$. We have the following table (Table 1).

Consequently, $\operatorname{pmin}_{f}(x) \neq \operatorname{pcl}_{f}(x)$ for every $x \in X$, hence $f$ is a topological pre-rough function on $X$.

A function $f:(X, \tau) \rightarrow(Y, \sigma)$ is said to be a topological pre-rough continuous at the point $x \in X$ if and only if $f\left({ }^{p} \overline{\operatorname{Seq}}(x)\right) \subseteq \operatorname{pcl}_{\sigma}(\operatorname{Seq}(f(x)))$, and it is a topological pre-rough continuous on $X$ if it is a topological pre-rough continuous at every point $x \in X$.

Example 3.2: Let $(X, \tau)$ and $(Y, \sigma)$ be topological spaces, where $X=\{a, b, c, d\}$ and $Y=\{1,2,3,4\}$ with $\tau=\{X, \varphi,\{a\},\{b\},\{a, b\},\{a, b, c\}\}$ and $\sigma=\{Y, \varphi,\{1\},\{1,4\},\{1,2,4\}\}$. Let $f:(X, \tau) \rightarrow(Y, \sigma)$ be a map defined by $f(a)=1, f(b)=2, f(c)=4$ and $f(d)=3$ (Table 2 ).

Consequently, $f\left({ }^{p} \overline{\operatorname{Seq}}(x)\right) \subset \operatorname{pcl}_{\sigma}(\operatorname{Seq}(f(x)))$ for every $x \in X$, hence $f$ is a topological pre-rough continuous function on $X$.

\section{The Pre-Approximations of Functions}

A function $f$ from $X$ to $Y$ is a relation from $X$ to $Y$ that satisfies:

1) $\operatorname{Dom}(f)=X$.

2) If $(x, y) \in f$ and $(x, z) \in f$, then $y=z$.

If $X=Y$, we say $f$ is a function on $X$. A function $f: X \rightarrow Y$ is completely represented by its graph $g(f)=\{(x, f(x)): x \in X\}$.

The concept of rough relations is defined by using a certain type of relation products. The following proposition

Table 1. $\operatorname{pmin}_{f}(x)$ and $\operatorname{pcl}_{f}(x)$ for some subsets of $X$.

\begin{tabular}{ccc}
\hline$X$ & $\operatorname{pmin}_{f}(x)$ & $p c l_{f}(x)$ \\
\hline$\{\mathrm{a}\}$ & $\{1,2,3\}$ & $\{3\}$ \\
$\{\mathrm{b}\}$ & $\{1\}$ & $\{1,3,4\}$ \\
$\{\mathrm{c}\}$ & $\mathrm{Y}$ & $\{4\}$ \\
$\{\mathrm{d}\}$ & $\{2\}$ & $\{2,3,4\}$ \\
\hline
\end{tabular}

Table 2. Topological pre-rough continuous function on $X$.

\begin{tabular}{ccccc}
\hline $\mathrm{X}$ & $\operatorname{Seq}(\mathrm{x})$ & ${ }^{p} \overline{\operatorname{Seq}}(x)$ & $f\left({ }^{p} \overline{\operatorname{Seq}}(x)\right)$ & $p c l_{\sigma}(\operatorname{Seq}(f(x)))$ \\
\hline$\{\mathrm{a}\}$ & $\{\mathrm{a}\}$ & $\{\mathrm{a}, \mathrm{c}, \mathrm{d}\}$ & $\{1,3,4\}$ & $\mathrm{Y}$ \\
$\{\mathrm{b}\}$ & $\{\mathrm{b}\}$ & $\{\mathrm{b}, \mathrm{c}, \mathrm{d}\}$ & $\{2,3,4\}$ & $\mathrm{Y}$ \\
$\{\mathrm{c}\}$ & $\{\mathrm{a}, \mathrm{b}, \mathrm{c}\}$ & $\mathrm{X}$ & $\mathrm{Y}$ & $\mathrm{Y}$ \\
$\{\mathrm{d}\}$ & $\{\mathrm{a}, \mathrm{b}, \mathrm{d}\}$ & $\mathrm{X}$ & $\mathrm{Y}$ & $\mathrm{Y}$ \\
\hline
\end{tabular}

will simplify the process of getting $\left(U_{1} \times U_{2}\right) /\left(R_{1} \times R_{2}\right)$ via $\left(U_{1} / R_{1}\right)$ and $\left(U_{2} / R_{2}\right)$.

Theorem 4.1: Let $A_{1}=\left(U_{1}, R_{1}\right)$ and $A_{2}=\left(U_{2}, R_{2}\right)$ be a pre-approximation spaces. Then we have $\left(U_{1} \times U_{2}\right) /\left(R_{1} \times R_{2}\right)=\left(U_{1} / R_{1}\right) \times\left(U_{2} / R_{2}\right)$.

Proof: Since for any $u, u^{\prime} \in U_{1}$ and $v, v^{\prime} \in U_{2}$, we have, $\left((u, v),\left(u^{\prime}, v^{\prime}\right)\right) \in R_{1} \cdot R_{2}$ iff $\left(u, u^{\prime}\right) \in R_{1}$ and $\left(v, v^{\prime}\right) \in R_{2}$. Let $[(u, v)]_{R_{1} \cdot R_{2}} \in\left(U_{1} \times U_{2}\right) /\left(R_{1} \times R_{2}\right)$.

Then we have

$$
\begin{aligned}
{[(u, v)]_{R_{1} \cdot R_{2}} } & =\left\{\left(\left(u^{\prime}, v^{\prime}\right):\left((u, v),\left(u^{\prime}, v^{\prime}\right)\right)\right) \in R_{1} \cdot R_{2}\right\} \\
& =\left\{\left(u^{\prime}, v^{\prime}\right):\left(u, u^{\prime}\right) \in R_{1} \text { and }\left(v, v^{\prime}\right) \in R_{2}\right\} \\
& =\left\{u^{\prime}:\left(u, u^{\prime}\right) \in R_{1}\right\} \times\left\{v^{\prime}:\left(v, v^{\prime}\right) \in R_{2}\right\} \\
& =[u]_{R_{1}} \times[v]_{R_{2}}
\end{aligned}
$$

Hence $\left(U_{1} \times U_{2}\right) /\left(R_{1} \times R_{2}\right)=\left(U_{1} / R_{1}\right) \times\left(U_{2} / R_{2}\right)$.

Let $f:\left(U_{1}, R_{1}\right) \rightarrow\left(U_{2}, R_{2}\right)$ be any function, where $A_{1}=\left(U_{1}, R_{1}\right)$ and $A_{2}=\left(U_{2}, R_{2}\right)$ are pre-approximation spaces, such that $R_{1}$ and $R_{2}$ are equivalence relations on $U_{1}$ and $U_{2}$ respectively. We define the equivalence relation $R=R_{1} \times R_{2}$ such that

$\left(U_{1} \times U_{2}\right) / R=\left(U_{1} / R_{1}\right) \times\left(U_{2} / R_{2}\right)$ is a partition of $U_{1} \times U_{2}$ for the function $g(f)=\left\{(x, f(x)): x \in U_{1}\right\}$ we define the pre-approximations

${ }_{p} \underline{R}(g(f))=\left\{\left(u_{1}, u_{2}\right) \in U_{1} \times U_{2}:\left[\left(u_{1}, u_{2}\right)\right]_{R} \subseteq g(f)\right\}$
${ }_{p} \underline{R}(g(f))=\left\{\left(u_{1}, u_{2}\right) \in U_{1} \times U_{2}:\left[\left(u_{1}, u_{2}\right)\right]_{R} \cap g(f) \neq \varphi\right\}$

A function $f: U_{1} \rightarrow U_{2}$ is said to be roughly in the pre-approximation space $A=\left(U^{2}, R\right)$, where $A_{1}=(U 1, R 1)$ and $A_{2}=\left(U_{2}, R_{2}\right)$ are pre-approximation spaces and $A=A_{1} \times A_{2}, U^{2}=U_{1} \times U_{2}$ if

$$
{ }_{p} \underline{R}(g(f)) \neq{ }^{p} \bar{R}(g(f)),
$$

otherwise $f$ is pre-exact function.

Example 4.1: Let $U 1=\{a, b, c, d, e\}$ and $U_{2}=\{1,2,3,4,5,6\}$ and consider the function $f: U_{1} \rightarrow U_{2}$ defined by $g(f)=\{(a, 3),(b, 6),(c, 5),(d, 2),(e, 3)\}$.

Consider the partitions $U_{1} / R_{1}=\{\{a, c\},\{b\},\{d, e\}\}$ and $U_{2} / R_{2}=\{\{1\},\{2,5\},\{3,4\},\{6\}\}$. Then

$$
\begin{aligned}
& \left(U_{1} \times U_{2}\right) / R=\left(U_{1} / R_{1}\right) \times\left(U_{2} / R_{2}\right) \\
= & \left\{\begin{array}{l}
\{(a, 1),(c, 1)\},\{(a, 2),(c, 2),(a, 5),(c, 5)\}, \\
\{(a, 3),(a, 4),(c, 3),(c, 4)\},\{(a, 6),(c, 6)\}, \\
\{(b, 1)\},\{(b, 2),(b, 5)\},\{(b, 3),(b, 4)\},\{(b, 6)\}, \\
\{(d, 1),(e, 1)\},\{(d, 2),(d, 5),(e, 2),(e, 5)\}, \\
\{(d, 3),(d, 4),(e, 3),(e, 4)\},\{(d, 6),(e, 6)\}
\end{array}\right\}
\end{aligned}
$$

is a partition of $\left(U_{1} \times U_{2}\right)$. 
Then $p \underline{R}(g(f))=\{(b, 6)\}$ and

${ }^{p} \bar{R}(g(f))=\left\{\begin{array}{l}(a, 3),(a, 4),(c, 3),(c, 4),(b, 6),(a, 2), \\ (c, 2),(a, 5),(c, 5),(d, 2),(d, 5),(e, 2), \\ (e, 5),(d, 3),(d, 4),(e, 3),(e, 4)\end{array}\right\}$

Therefore the function $f$ is a rough function such that ${ }_{p} \underline{R}(g(f)) \neq^{p} \bar{R}(g(f))$.

For the function $f: U_{1} \rightarrow U_{2}$, we observe that in general ${ }^{p} \bar{R}(g(f))$ and ${ }_{p} \underline{R}(g(f))$ are not functions from $U 1$ into $U 2$. We point that, the process of defining an pre-approximations on $(U 1 \times U 2)$ such that ${ }^{p} \bar{R}(g(f))$ and $p \underline{R}(g(f))$ are functions is an open question to be solved in our next work.

Theorem 4.2: For every function $f: U_{1} \rightarrow U_{2}$ such that $A_{1}=\left(U_{1}, R_{1}\right)$ and $A_{2}=\left(U_{2}, R_{2}\right)$ are selective preapproximation spaces then $f$ is an pre-exact function.

Proof: Since in any selective pre-approximation space, $[(x, y)]=\{(x, y)\}$ then ${ }^{p} \bar{R}(g(f))={ }_{p} \underline{R}(g(f))$ then $f$ is an preexact function.

Example 4.2: Let $U_{1}=\{a, b, c, d\}$ and $U 2=\{1,2,3\}$. Consider the function $f: U 1 \rightarrow U 2$, defined by $g(f)$ $=\{(a, 1),(b, 1),(c, 2),(d, 3)\}$ and consider the partitions $U_{1} / R_{1}=\{\{a\},\{b\},\{c\},\{d\}\}$ and $U_{2} / R_{2}=\{\{1\},\{2\},\{3\}\}$. Then

$$
\begin{aligned}
& U_{1} \times U_{2} / R=\left(U_{1} / R_{1}\right) \times\left(U_{2} / R_{2}\right)= \\
& \left\{\begin{array}{l}
\{(a, 1)\},\{(a, 2)\},\{(a, 3)\},\{(b, 1)\},\{(b, 2)\},\{(b, 3)\}, \\
\{(c, 1)\},\{(c, 2)\},\{(c, 3)\},\{(d, 1)\},\{(d, 2)\},\{(d, 3)\}
\end{array}\right\}
\end{aligned}
$$

is a partition of $U 1 \times U 2$.

Then $p \underline{R}(g(f))=\{(a, 1),(b, 1),(c, 2),(d, 3)\}$ and ${ }^{p} \bar{R}(g(f))=\{(a, 1),(b, 1),(c, 2),(d, 3)\}$, then $f$ is an pre-exact function.

For a function $f: U 1 \rightarrow U 2$ such that $A_{1}=(U 1, R 1)$ and $A_{2}=\left(U_{2}, R_{2}\right)$ are selective pre-approximation spaces then

1) If $f$ is a one-to-one function then also both ${ }^{p} \bar{R}(g(f))$ and ${ }_{p} \underline{R}(g(f))$.

2) If $f$ is onto function then also both ${ }^{p} \bar{R}(g(f))$ and ${ }_{p} \underline{R}(g(f))$.

3) If $f$ is a pre-continuous function then also both ${ }^{p} \bar{R}(g(f))$ and $p \underline{R}(g(f))$.

No function $f: U_{1} \rightarrow U_{2}$ such that $A_{1}=\left(U_{1}, R_{1}\right)$ and $A_{2}=\left(U_{2}, R_{2}\right)$ are not selective approximation spaces is pre-exact, and $f$ is not a constant function.

\section{An Alternative Description of Topological Pre-Rough Functions}

Let $\left(U_{1}, \tau_{1}\right)$ and $\left(U_{2}, \tau_{2}\right)$ be any topological spaces, the function $f:(U 1, \tau 1) \rightarrow\left(U_{2}, \tau_{2}\right)$, can be considered as a relation of $U_{1} \times U_{2}$ and if $\beta$ is a basis of $\tau_{1}$ and $\beta_{2}$ is a basis of $\tau_{2}$, then $\beta=\beta_{1} \times \beta_{2}$ is a basis of the topology $\tau$ on $U_{1} \times U_{2}$. In the topology $\left(U_{1} \times U_{2}, \tau\right)$ we define ${ }_{p} \underline{f}=\operatorname{pint}(f)$ and ${ }^{p} \bar{f}=\operatorname{pcl}(f)$ for the function $f$. Let $f:\left(U_{1}, \tau_{1}\right) \rightarrow\left(U_{2}, \tau_{2}\right)$ be a function, where $\left(U_{1}, \tau_{1}\right)$ and $\left(U_{2}, \tau_{2}\right)$, are topological spaces, the function $f$ is called a topological pre-rough function in $\left(U_{1} \times U_{2}, \tau\right)$ iff ${ }_{p} f \neq^{p} \bar{f}$ otherwise, $f$ is an preexact function in $\left(U_{1} \times U_{2}, \tau\right)$.

Example 5.1: Let $\left(U_{1}, \tau_{1}\right)$ and $\left(U_{2}, \tau_{2}\right)$ be any topological spaces where $U_{1}=(a, b, c)$,

$U_{2}=\{1,2,3,4\}, \tau_{1}=\left\{U_{1}, \varphi,\{a\},\{b, c, d\}\right\}$,

$\tau_{2}=\left\{U_{2}, \varphi,\{3\},\{1,2,4\}\right\}$ Consider $\beta_{1}=\{\{a\},\{b, c, d\}\}$ and $\beta_{2}=\{\{3\},\{1,2,4\}\}$ are basis of $\tau_{1}$ and $\tau_{2}$ respectively. Let $f: U_{1} \rightarrow U_{2}, g: U_{1} \rightarrow U_{2}$ and $h: U_{1} \rightarrow U_{2}$ are mappings defined by

$f=\{(a, 3),(b, 1),(c, 2),(d, 4)\}$,

$g=\{(a, 2),(b, 3),(c, 1),(d, 4)\}$

and $h=\{(a, 3),(b, 3),(c, 3),(d, 3)\}$.

Then ${ }_{p} f=\operatorname{pinf}(f)=\{(a, 3)\}$ and

${ }^{p} \bar{f}=c l(f)=\left\{\begin{array}{l}(a, 3),(b, 1),(b, 2),(b, 4),(c, 1), \\ (c, 2),(c, 4),(d, 1),(d, 2),(d, 4)\end{array}\right\}$

Then $f$ is a pre-rough function in $\left(U_{1} \times U_{2}, \tau\right)$. Also, ${ }_{p} \underline{g}=\operatorname{pint}(g)=\varphi$ and ${ }^{p} \bar{g}=p c l(g)=U_{1} \times U_{2}$

We call $g$ is a function not defined from pre-lower and from upper. Finally, for the constant function $h$, we have ${ }_{p} \underline{h}=\operatorname{pint}(h)=\operatorname{pcl}(h)={ }^{p} \bar{h}$, and $h$ is an pre-exact function. In fact, $h$ is the only exact function in $\left(U_{1} \times U_{2}, \tau\right)$.

According to Example 1, we have the following:

1) The function $f$ is continuous, but ${ }_{p} f$ and ${ }^{p} \bar{f}$ are not functioning, hence we cannot say that $f$ or $f$ is pre-continuous.

2) The function $h$ is always precontinuous function, and it is an pre-exact function, hence ${ }_{p} \underline{h}$ and ${ }^{p} \bar{h}$ is pre-continuous functions.

\section{Experiments and Evaluations}

This section shows the effectiveness of using pre-rough functions for extracting new data from multi-valued information systems.

In this section, we briefly describe the Rheumatic Fever datasets mentioned in [37] as a topological application of rough functions. As mentioned in [39] rheumatic fever is a very common disease and it has many symptoms differs from patient to another though the diagnosis is the same. So, we obtained the following example on four rheumatic fever patients. All patients are between 9-12 years old with a history of Arthurian began from 
age 3-5 years. This disease has many symptoms and it is usually started in young age and still with the patient along his life.

Table 3 in [37] introduced the seven patients characterized by 8 symptoms (attributes) using them to decide the diagnosis for each patient (decision attribute). Where the attributes are satisfied in Table 2 in [37].

We recall and sell it here Table 3.

If we defined the following mapping on Table $\mathbf{3}$ :

$f: U \rightarrow P(U):$

$f(p 1)=\{p 1, p 2\}, f(p 2)=\{p 2, p 3\}$,

$f(p 3)=\{p 3\}, f(p 4)=\{p 2, p 4\}, f(p 5)=\{p 1, p 5, p 7\}$

$f(p 6)=\{p 6\}, f(p 7)=\{p 5, p 7\}$

From the relation $R_{a}=\left\{(x, y): f_{a}(x) \subseteq f_{a}(y)\right\}$ where $a$ is an element of the power set of the set of condition attributes $\{\alpha, \beta, \delta\}$. The the following classes $\chi 1=\left\{x R_{a}: x \in U\right\}$ and $\chi 2=\left\{R_{a} x: x \in U\right\}$ are two subbases of two topologies on $\mathrm{U}$ such that $R_{a} x=\left\{y: y R_{a} x\right\}$. Then according to Table 3 we have the following couples of topologies:

$$
\begin{aligned}
\tau_{1}^{\alpha}=\left\{\begin{array}{l}
U, \varphi,\left\{p_{2}\right\},\left\{p_{3}\right\},\left\{p_{2}, p_{3}\right\},\left\{p_{1}, p_{2}\right\}, \\
\left\{p_{1}, p_{2}, p_{3}\right\},\left\{p_{2}, p_{3}, p_{4}, p_{5}, p_{6}, p_{7}\right\}, \\
\left\{p_{1}, p_{2}, p_{4}, p_{5}, p_{6}, p_{7}\right\},\left\{p_{2}, p_{4}, p_{5}, p_{6}, p_{7}\right\}
\end{array}\right\}, \\
\tau_{2}^{\alpha}=\left\{\begin{array}{l}
U, \varphi,\left\{p_{1}\right\},\left\{p_{3}\right\},\left\{p_{1}, p_{3}\right\},\left\{p_{4}, p_{5}, p_{6}, p_{7}\right\}, \\
\left\{p_{3}, p_{4}, p_{5}, p_{6}, p_{7}\right\},\left\{p_{1}, p_{4}, p_{5}, p_{6}, p_{7}\right\}, \\
\left\{p_{1}, p_{2}, p_{4}, p_{5}, p_{6}, p_{7}\right\},\left\{p_{2}, p_{3}, p_{4}, p_{5}, p_{6}, p_{7}\right\}
\end{array}\right\} \\
\tau_{1}^{\beta}=\left\{\begin{array}{l}
U, \varphi,\left\{p_{5}\right\},\left\{p_{7}\right\},\left\{p_{3}, p_{7}\right\},\left\{p_{1}, p_{4}\right\}, \\
\left.\left\{p_{5}, p_{7}\right\},\left\{p_{3}, p_{5}, p_{7}\right\},\left\{p_{1}, p_{4}, p_{5}\right\},\right\} \\
\left\{p_{1}, p_{4}, p_{5}, p_{7}\right\},\left\{p_{1}, p_{2}, p_{4}, p_{6}\right\}
\end{array}\right\} \\
\tau_{2}^{\beta}=\left\{\begin{array}{l}
U, \varphi,\left\{p_{3}, p_{5}, p_{7}\right\},\left\{p_{2}, p_{3}, p_{6}\right\},\left\{p_{2}, p_{3}, p_{6}, p_{7}\right\}, \\
\left\{p_{1}, p_{2}, p_{4}, p_{6}\right\},\left\{p_{1}, p_{2}, p_{3}, p_{4}, p_{6}\right\}, \\
\left\{p_{2}, p_{3}, p_{5}, p_{6}, p_{7}\right\},\left\{p_{1}, p_{2}, p_{4}, p_{5}, p_{6}\right\}, \\
\left\{p_{1}, p_{2}, p_{3}, p_{4}, p_{5}, p_{6}\right\},\left\{p_{1}, p_{2}, p_{3}, p_{4}, p_{6}, p_{7}\right\}
\end{array}\right\} \\
\tau_{1}^{\delta}=\left\{\begin{array}{l}
U, \varphi,\left\{p_{4}\right\},\left\{p_{1}, p_{3}, p_{6}\right\},\left\{p_{1}, p_{3}, p_{4}, p_{6}\right\}, \\
\left\{p_{1}, p_{3}, p_{5}, p_{6}\right\},\left\{p_{1}, p_{2}, p_{3}, p_{6}, p_{7}\right\}, \\
\left\{p_{1}, p_{2}, p_{3}, p_{5}, p_{6}, p_{7}\right\},\left\{p_{1}, p_{3}, p_{4}, p_{5}, p_{6}\right\}, \\
\left\{p_{1}, p_{2}, p_{3}, p_{4}, p_{6}, p_{7}\right\} \\
\left\{p_{1}, p_{2}, p_{3}, p_{7}\right\},\left\{p_{5}\right\},\left\{p_{2}, p_{7}\right\},\left\{p_{4}, p_{5}\right\},
\end{array}\right\} \\
\left\{\begin{array}{l}
\left.U, p_{5}, p_{7}\right\},\left\{p_{2}, p_{4}, p_{5}, p_{7}\right\}, \\
U
\end{array}\right\}
\end{aligned}
$$

$$
\begin{aligned}
& \tau_{2}^{\alpha \beta}=\tau_{2}^{\alpha} \cap \tau_{2}^{\beta}=\{U, \varphi\} \\
& \tau_{1}^{\alpha \delta}=\tau_{1}^{\alpha} \cap \tau_{1}^{\delta}=\{U, \varphi\} \\
& \tau_{2}^{\alpha \delta}=\tau_{2}^{\alpha} \cap \tau_{2}^{\delta}=\{U, \varphi\} \\
& \tau_{1}^{\beta \delta}=\tau_{1}^{\beta} \cap \tau_{1}^{\delta}=\left\{U, \varphi,\left\{p_{5}\right\}\right\} \\
& \tau_{2}^{\beta \delta}=\tau_{2}^{\beta} \cap \tau_{2}^{\delta}=\left\{U, \varphi,\left\{p_{1}, p_{2}, p_{3}, p_{4}, p_{6}, p_{7}\right\}\right\} \\
& \tau_{1}^{\alpha \beta \delta}=\tau_{1}^{\alpha} \cap \tau_{1}^{\beta} \cap \tau_{1}^{\delta}=\{U, \varphi\} \\
& \tau_{2}^{\alpha \beta \delta}=\tau_{2}^{\alpha} \cap \tau_{2}^{\beta} \cap \tau_{2}^{\delta}=\{U, \varphi\}
\end{aligned}
$$

According to the mapping $f: U \rightarrow P(U)$ and using each one of the above topologies we can deduce that the decision topology can be given by:

$$
\tau_{D}=\left\{U, \phi,\left\{p_{1}, p_{2}, p_{3}, p_{6}, p_{7}\right\},\left\{p_{4}\right\},\left\{p_{5}\right\},\left\{p_{4}, p_{5}\right\}\right. \text {. }
$$

Now we can construct a familiar system of Table $\mathbf{3}$ contains only the pre-rough images constructed using the terminology of pre-rough functions. This system can be the reduction system of Table 3 and it given in Table 4.

This means that we can remove the conditional attribute $\{\alpha\}$ without any loss of information.

\section{Conclusions}

We conclude that the emergence of topology and its operators $[38,39]$ in the construction of some rough set concepts will help to get rich results that yields a lot of logical statements which discover hidden relations between data and moreover, probably help in producing

Table 3. Multi-valued information system of [37].

\begin{tabular}{ccccc}
\hline $\mathrm{D}$ & $\delta$ & $\beta$ & $\alpha$ & $\mathrm{U}$ \\
\hline $\mathrm{p}_{1}$ & $\left\{\alpha_{2}\right\}$ & $\left\{\beta_{1}, \beta_{2}, \beta_{4}\right\}$ & $\left\{\delta_{1}\right\}$ & $\left\{d_{3}\right\}$ \\
$\mathrm{p}_{2}$ & $\left\{\alpha_{1}, \alpha_{2}\right\}$ & $\left\{\beta_{1}, \beta_{2}\right\}$ & $\left\{\delta_{1}, \delta_{3}\right\}$ & $\left\{d_{3}\right\}$ \\
$\mathrm{p}_{3}$ & $\left\{\alpha_{3}\right\}$ & $\left\{\beta_{1}, \beta_{3}\right\}$ & $\left\{\delta_{1}\right\}$ & $\left\{\mathrm{d}_{3}\right\}$ \\
$\mathrm{p}_{4}$ & $\left\{\alpha_{1}\right\}$ & $\left\{\beta_{1}, \beta_{2}, \beta_{4}\right\}$ & $\left\{\delta_{4}\right\}$ & $\left\{\mathrm{d}_{1}\right\}$ \\
$\mathrm{p}_{5}$ & $\left\{\alpha_{1}\right\}$ & $\left\{\beta_{5}\right\}$ & $\left\{\delta_{1}, \delta_{2}\right\}$ & $\left\{\mathrm{d}_{2}\right\}$ \\
$\mathrm{p}_{6}$ & $\left\{\alpha_{1}\right\}$ & $\left\{\beta_{1}, \beta_{2}\right\}$ & $\left\{\delta_{1}\right\}$ & $\left\{d_{3}\right\}$ \\
$\mathrm{p}_{7}$ & $\left\{\alpha_{1}\right\}$ & $\left\{\beta_{1}, \beta_{3}, \beta_{4}\right\}$ & $\left\{\delta_{1}, \delta_{3}\right\}$ & $\left\{d_{3}\right\}$ \\
\hline
\end{tabular}

Table 4. Reduced System.

\begin{tabular}{ccc}
\hline $\mathrm{U}$ & $\beta$ & $\delta$ \\
\hline $\mathrm{p}_{1}$ & $\left\{\beta_{1}, \beta_{2}, \beta_{4}\right\}$ & $\left\{\delta_{1}\right\}$ \\
$\mathrm{p}_{2}$ & $\left\{\beta_{1}, \beta_{2}\right\}$ & $\left\{\delta_{1}, \delta_{3}\right\}$ \\
$\mathrm{p}_{3}$ & $\left\{\beta_{1}, \beta_{3}\right\}$ & $\left\{\delta_{1}\right\}$ \\
$\mathrm{p}_{4}$ & $\left\{\beta_{1}, \beta_{2}, \beta_{4}\right\}$ & $\left\{\delta_{4}\right\}$ \\
$\mathrm{p}_{5}$ & $\left\{\beta_{5}\right\}$ & $\left\{\delta_{1}, \delta_{2}\right\}$ \\
$\mathrm{p}_{6}$ & $\left\{\beta_{1}, \beta_{2}\right\}$ & $\left\{\delta_{1}\right\}$ \\
$\mathrm{P}_{7}$ & $\left\{\beta_{1}, \beta_{3}, \beta_{4}\right\}$ & $\left\{\delta_{1}, \delta_{3}\right\}$ \\
\hline
\end{tabular}


accurate programs. These topological operators will play an essential role in data mining and knowledge discovery in databases. In this paper, we give an overview of several dissipated results on the pre-rough functions. More specifically, we attempt to show: usefulness of this new concept in a calculus of rough functions.

The future application of this work will be useful in many fields such as Fuzzy Expert Systems [40] by generalizations of rough functions for fuzzy rough functions. It also is useful in knowledge discovery methods [41].

\section{REFERENCES}

[1] Z. Pawlak, "Rough Sets," International Journal of Parallel Programming, Vol. 11, No. 5, 1982, pp. 341-356. doi:10.1007/BF01001956

[2] X. Wang, E. C. C. Tsang, S. Zhao, D. Chen and S. Yeung, "Learning Fuzzy Rules from Fuzzy Samples Based on Rough Set Technique," Information Sciences, Vol. 177, No. 15, 2007, pp. 4493-4514. doi:10.1016/j.ins.2007.04.010

[3] S. Zhao and E. C. C. Tsang, "On Fuzzy Approximation Operators in Attribute Reduction with Fuzzy Rough Sets," Information Sciences, Vol. 178, No. 16, 2008, pp. 3163-3176.

[4] L. A. Zadeh, "Fuzzy Sets," Information and Control, Vol. 8, No. 3, 1965, pp. 338-353. doi:10.1016/S0019-9958(65)90241-X

[5] Z. Bonikowski, "Algebraic Structures of Rough Sets," In: W. Ziarko, Ed., Rough Sets, Fuzzy Sets and Knowledge Discovery, Springer, London, 1994, pp. 243-247. doi:10.1007/978-1-4471-3238-7 29

[6] E. Bryniaski, "A Calculus of Rough Sets of The First Order," Bulletin of the Polish Academy of Sciences, Vol. 16, 1989, pp. 71-77.

[7] Y. Y. Yao, "Constructive and Algebraic Methods of Theory of Rough Sets," Information Sciences, Vol. 109, No. 1-4, 1998, pp. 21-47. doi:10.1016/S0020-0255(98)00012-7

[8] A. Skowron and J. Stepaniuk, "Tolerance Approximation Spaces," Fund Information, Vol. 27, No. 2-3, 1996, pp. 245-253.

[9] E. F. Lashin, A. M. Kozae, A. A. Abo Khadra and T. Medhat, "Rough Set Theory for Topological Spaces," International Journal of Approximate Reasoning, Vol. 40, No. 1-2, 2005, pp. 35-43. doi:10.1016/j.ijar.2004.11.007

[10] K. Qin and Z. Pei, "On the topological Properties of Fuzzy Rough Sets," Fuzzy Sets and Systems, Vol. 151, No. 3, 2005, pp. 601-613. doi:10.1016/j.fss.2004.08.017

[11] A. Wasilewska, “Topological Rough Algebras," In: T. Y. Lin and N. Cercone, Eds., Rough Sets and Data Mining, Kluwer Academic Publishers, Boston, 1997, pp. 425-441. doi:10.1007/978-1-4613-1461-5 21

[12] W. Zhu, "Topological Approaches to Covering Rough Sets," Information Sciences, Vol. 177, No. 15, 2007, pp. 1499-1508. doi:10.1016/j.ins.2006.06.009

[13] T. J. Li, Y. Leung and W. X. Zhang, "Generalized Fuzzy
Rough Approximation Operators Based on Fuzzy Coverings," International Journal of Approximate Reasoning, Vol. 48, No. 3, 2008, pp. 836-856. doi:10.1016/j.ijar.2008.01.006

[14] R. Biswas, "On Rough Sets and Fuzzy Rough Sets," Bulletin of the Polish Academy of Sciences, Vol. 42, 1992, pp. 343-349.

[15] G. Liu, "Generalized Rough Sets over Fuzzy Lattices," Information Sciences, Vol. 178, No. 6, 2008, pp. 16511662. doi:10.1016/j.ins.2007.11.010

[16] A. M. Rolka and L. Rolka, "Fuzzy Rough Approximations of Process Data," International Journal of Approximate Reasoning, Vol. 49, No. 2, 2008, pp. 301-315. doi:10.1016/j.ijar.2007.03.016

[17] M. Banerjee and S. K. Pal, "Roughness of a Fuzzy Set," Information Sciences, Vol. 93, No. 3-4, 1995, pp. 235246. doi:10.1016/0020-0255(96)00081-3

[18] R. Biswas, "On Rough Fuzzy Sets," Bulletin of the Polish Academy of Sciences, Vol. 42, 1994, pp. 352-355.

[19] D. Dubois and H. Prade, "Rough Fuzzy Sets and Fuzzy Rough Sets," International Journal of General Systems, Vol. 17, No. 2-3, 1990, pp. 191-208. doi:10.1080/03081079008935107

[20] C. Degang, Y. Wenxia and Li Fachao, "Measures of General Fuzzy Rough Sets on a Probabilistic Space," Information Sciences, Vol. 178, No. 16, 2008, pp. 3177-3187. doi:10.1016/j.ins.2008.03.020

[21] Z. Gong, B. Sun and D. Chen, "Rough Set Theory for the Interval-Valued Fuzzy Information Systems," Information Sciences, Vol. 178, No. 8, 2008, pp. 1968-1985. doi:10.1016/j.ins.2007.12.005

[22] Y. Yang and C. Hinde, "A New Extension of Fuzzy Sets Using Rough Sets: R-Fuzzy Sets," Information Sciences, Vol. 180, No. 3, 2010, pp. 354-365. doi:10.1016/j.ins.2009.10.004

[23] P. Bhattacharya and B. K. Lahiri, "Semi Generalized Closed Sets in Topology," Indian Journal of Mathematics, Vol. 29, 1987, pp. 373-382.

[24] A. Skowron, "On Topology Information Systems," Bulletin of the Polish Academy of Sciences, Vol. 3, 1989, pp. 87-90.

[25] Z. Pawlak, "Rough Sets: Theoretical Aspects of Reasoning about Data," Kluwer Academic Publishers, Boston, 1991.

[26] A. S. Mashhour, M. E. Abd El-Monsef and S. N. El-Deeb, "On Pre-Continuous and Weak Pre Continuous Mappings," Mathematical Physical and Engineering Sciences, Vol. 53, 1982, pp. 47-53.

[27] O. Najsted, "On Some Classes of Nearly Open Sets," Pacific Journal of Mathematics, Vol. 15, 1965, pp. 961970.

[28] J. R. Munkres, “Topology, a First Course,” Prentice-Hall, Upper Saddle River, 1975.

[29] M. E. Abd El-Monsef, S. N. El-Deeb and R. A. Mahmoud, " $\beta$-Open Sets and $\beta$-Continuous Mappings," Bulletin of the Faculty of Science, Assiut University, Asyut, 1983. 
[30] D. Andrijevic, "On b-Open Sets," Matematicki Vesnik, Vol. 48, 1996, pp. 59-64.

[31] A. S. Mashhour, I. A. Hasanein and S. N. El Deeb, "A Note on $\alpha$-Continuous and $\alpha$-Open Mappings," Acta Mathematica Hungarica, Vol. 41, No. 3-4, 1983, pp. 213218.

[32] J. Kortelainen, "On the Relationship between Modified Sets, Topological Spaces and Rough Sets," Fuzzy Sets and Systems, Vol. 61, No. 1, 1994, pp. 91-95. doi:10.1016/0165-0114(94)90288-7

[33] Y. Y. Yao, "Two Views of the Theory of Rough Sets in Finite Universes," International Journal of Approximate Reasoning, Vol. 15, No. 4, 1996, pp. 291-317. doi:10.1016/S0888-613X(96)00071-0

[34] Y. Y. Yao and T. Y. Lin, "Generalization of Rough Sets Using Modal Logics," Intelligent Automation \& Soft Computing, Vol. 2, 1996, pp. 103-120.

[35] Z. Pawlak, "On Rough Relations," Bulletin of the Polish Academy of Sciences, Vol. 34, 1986, pp. 9-10.

[36] Z. Pawlak, "Rough Sets, Rough Relations and Rough Functions," Bull of the Polish Academy of Sciences, Vol.
13, 1996, pp. 15-19.

[37] A. S. Salama, "Bitopological Rough Approximations with Medical Applications," Journal of King Saud University (Science), Vol. 22, No. 3, 2010, pp. 177-183. doi:10.1016/j.jksus.2010.04.010

[38] J. Kelley, "General Topology," Van Nostrand Company, New York, 1955.

[39] N. Levine, "Semi Open Sets and Semi Continuous Mappings in Topological Spaces," American Mathematical Monthly, Vol. 70, No. 1, 1963, pp. 36-41. doi: $10.2307 / 2312781$

[40] M. H. F. Zarandi, et al., "A Fuzzy Expert System Architecture for Intelligent Tutoring Systems: A Cognitive Mapping Approach," Journal of Intelligent Learning Systems and Applications, Vol. 4, 2012, pp. 29-40. doi:10.4236/jilsa.2012.41003

[41] G. Serpen and M. Riesen "Knowledge Discovery for Query Formulation for Validation of a Bayesian Belief Network," Journal of Intelligent Learning Systems and Applications, Vol. 2, No. 3, 2010, pp. 156-166. 